\title{
DEVELOPMENT AND TESTING OF LOWER LIMB EXOSKELETON FOR STROKE PATIENTS
}

\author{
Diloshini Devi, Selva Kumar \\ Department of Instrumentation Engineering, \\ St. Joseph's Engineering College, Chennai, Tamil Nadu
}

\begin{abstract}
The supply of blood flow to the brain is either reduced or blocked this leads to stroke due to lack of oxygen and nutrients to the brain cells. The post-stroke symptoms include irregular gait patterns, deviation from normal gait movement so the system will help to normalize the pattern of patients. A device to be designed which goes along well with natural human gait for translational of body center of mass with minimum expenditure of energy. This is possible when developing mechanism in which knee, ankle, foot motion coordinating and interacting with each other. The system should support stability, mobility and maintain balance along CoM. This mechanism is simple but it is limited adaption with certain gait condition and imitates knee during swing and support phase referred as bio imitation. The main considerations include Force, Torque, Position and bioelectrical signals. Walking is derived as sum of gravitational potential and forward kinetic energy, and thus energy is exchanged in terms of walking. Human walking is categorized as example of Inverted pendulum model. A prototype model of exoskeleton is modelled to move in a desired position with zero impedance.
\end{abstract}

Key Words: Gait pattern, Centre of mass, bio imitation and inverted pendulum model, impedance.

\section{INTRODUCTION}

The enhancement of motor cortex representation recovers and strengthen muscle to support interaction between various lower limb joints. It provides external torque and sensors are used to measure angle of torso, shift in gravity and estimate user's walking intention. Variation of gait phase in uphill and downhill have same radius but change in orientation. The system is powered by battery to adjust neutral angle of foot. The two main goal in determining of gait includes to reduce maximum height of body center of mass during midstance and to increase minimum height of body $\mathrm{CoM}$ at heel strike and toe off. The length and leg force so as to maintain constant height of center of mass. Humans adjust their foot and ankle motion to achieve ankle foot rollover shape radius. Actuation of ankle, knee and foot to produce torque and to support in ground to elevation. In the passive device neutral angle of ankle is fixed. The neutral angle changes when sitting and standing.

The main constraints in biological design which states that Minimum weight and energy consumption, Minimum load to tissue, multi functionality for human limbs and induvial select a range of speed and motion. To increase the efficiency of the system integration of mechanical, electrical and control system is essential. The control system function as MasterSlave configuration. Actuation of knee, ankle and hip joint is done by motors. Measurements of ground reaction forces and torque are calculated with help of sensors.

\section{ACTUATION JOINTS}

Knee flexion occurs after heel strike and extends up to mid stance and to achieve ground clearance. CoM needs to be positioned vertically above stance foot for stability. Knee flexion is approximately 15 degrees at normal speed. In case of stroke patients, it fails to collect sensory information for the locomotion. When knee joint moves in forward direction the muscles contract and loosen and ligaments extend and shrink. Walking constitutes repetition of single and double support. Tendons will store the elastic energy. In human motor system muscles will be considered as actuators.

The system is used to read EMG pattern and produce desirable torque based on the reading. Absolute value of work to turn the leg is proportional to the square of natural frequency and the angle to drive it. Hip joint should be back drivable. It monitors ankle joint and reacts when its angle exceeds certain value. The outside leg take farther radius circle whereas inside leg takes small radius. The role actuation of knee includes absorption of shock in the system and knee assistance to full extension of knee.

$$
\mathrm{T}=\mathrm{K} 1 \alpha \text { stance }
$$

\section{INVETERD PENDULUM MODEL}

The best illustration for inverted pendulum model is Human gait. It includes six determinants of gait
a. Pelvic rotation
b. Pelvic Tilt
c. Knee flexion
d. Foot and Knee mechanism-1
e. Foot and knee mechanism-2
f. Lateral pelvis displacement

Pelvic tilt is rotation in coronal plane about anterior or posterior axis and its approx. 5 degree at normal speed. It requires higher degree of freedom and high power and mass. 


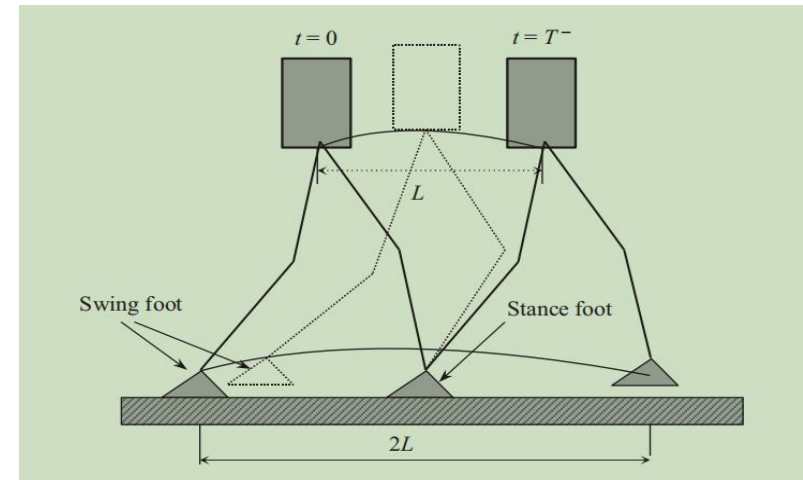

Fig.1. Inverse pendulum model at $\mathrm{t}=0$ and $\mathrm{t}=\mathrm{T}$

\section{COORDINATION OF PROCESS}

The commands provided by EMG signals is used to derive movement in human limbs and also computes biofeedback. Its function includes propulsion and weight bearing. The system includes error management function, Acquisition system is placed on the user for data collection and data analysis.

$\mathrm{Y}_{\mathrm{i}}=\mathrm{Q}_{\mathrm{i}}-\mathrm{H}_{\mathrm{d}, \mathrm{i}}(\theta)$

Where,

$\mathrm{Q}_{\mathrm{i}}=$ actual angle,

$\mathrm{H}_{\mathrm{d}, \mathrm{i}}(\theta)=$ desired angle

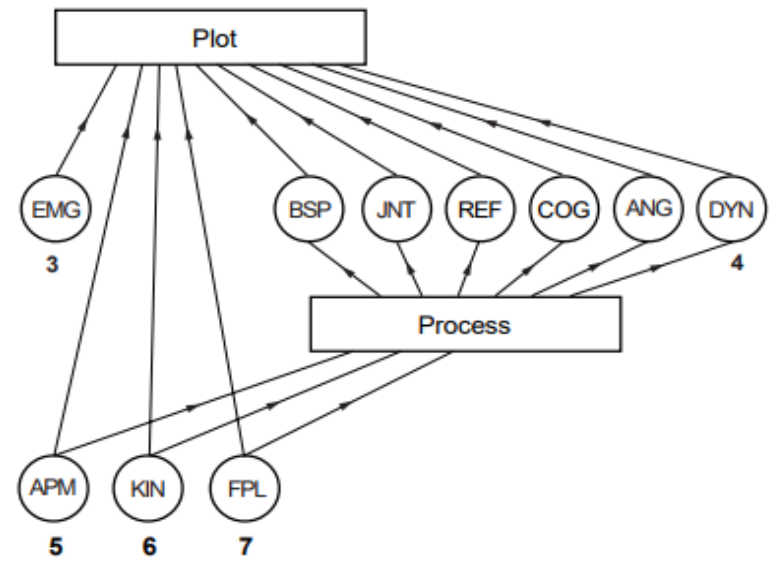

Fig.2. State diagram to coordinate various stages of process

\section{GAIT PHASE}

Walking and running can be portraited as distinguished periodic orbits of mechanical systems that are highly nonlinear and hybrid. Walking cycle is periodic phases of swing phase and impact phase.

Gait phase commences at time $t=0$ with one foot, called the swing foot, leaving the ground Then, over a period $T$, the swing foot moves forward a distance $2 L$ and the hip a distance $L$. Gait phase finishes at time $t=T$ then the single support will be in contact with the ground. Throughout this period, the other foot, called the stance foot, remains fixed on the ground, thus providing a single support for stability.

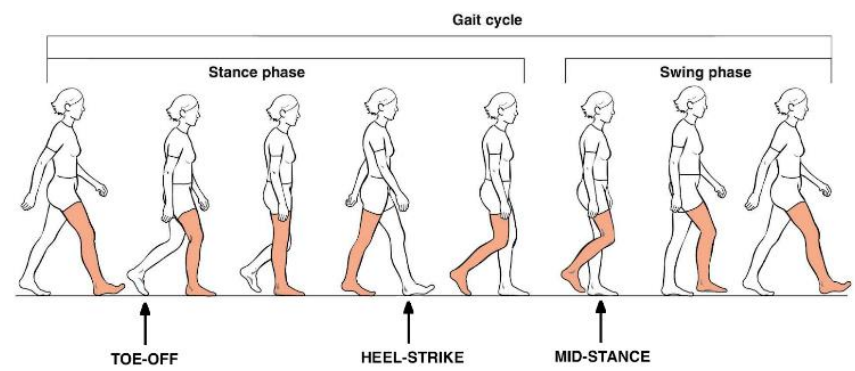

Fig.3. Inverse pendulum model at $\mathrm{t}=0$ and $\mathrm{t}=\mathrm{T}$

At a given instant during the single-support phase, the position of the bipedal robot relative to the stance foot can be specified by the following coordinate vector:

$$
\mathrm{k}=\left[\begin{array}{lllll}
\psi & \alpha 1 & \alpha 2 & \beta & \beta
\end{array}\right]
$$

Motors located at the robot's hip, knees and stance foot generate torques $\tau 1$ to $\tau 5$. These can be grouped into vector $\tau$ where

$$
\boldsymbol{\tau}=\left[\begin{array}{lllll}
\tau 1 & \tau 2 & \tau 3 & \tau 4 & \tau 5
\end{array}\right]
$$

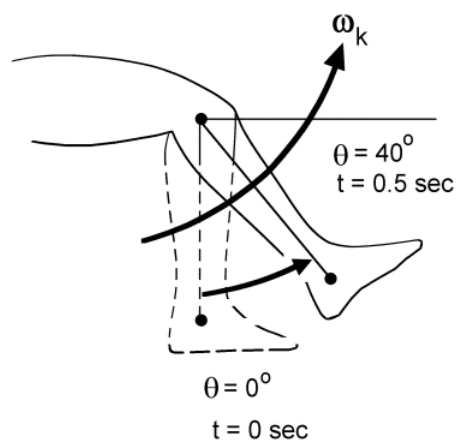

Fig.4. State diagram to coordinate various stages of process

\section{RESULTS}

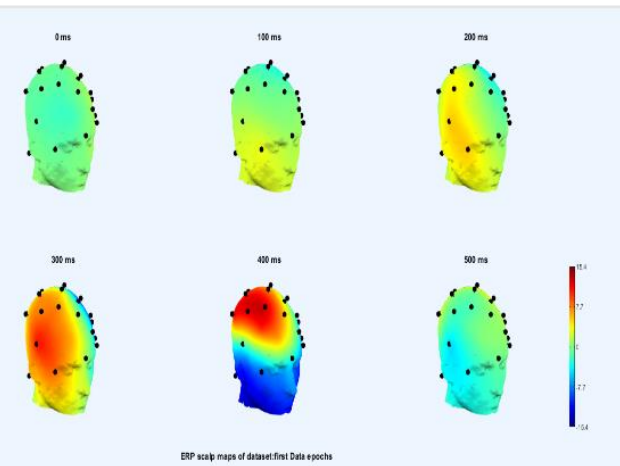

Fig.4. Brain signals analysed using MATLAB 


\section{International Journal of Engineering Applied Sciences and Technology, 2020 \\ Vol. 4, Issue 12, ISSN No. 2455-2143, Pages 423-425 \\ Published Online April 2020 in IJEAST (http://www.ijeast.com)}

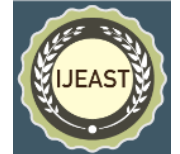

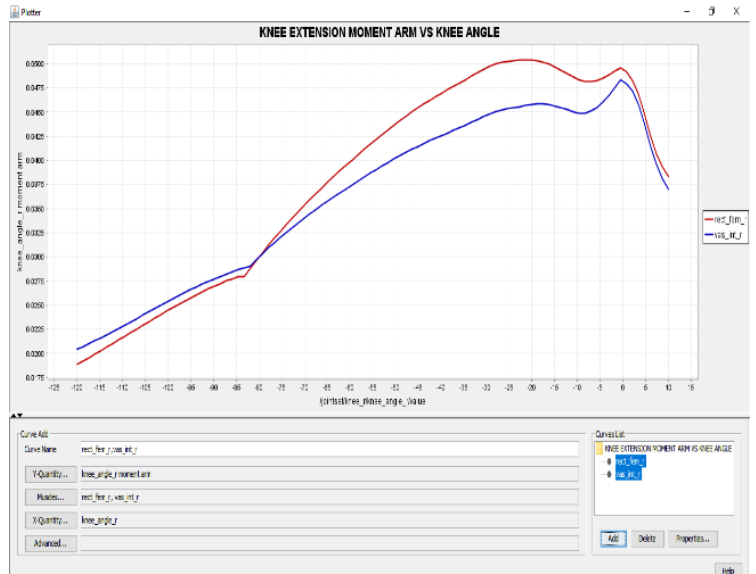

Fig.5.Knee Extension and Knee angle for sample data Using opensim

\section{CONCLUSION}

Wearable exoskeletons are promising technology in rehabilation field.This prototype model provides desirable torue without much energy expenditure.Battery technology and actuator technologies should be designed to meet the power and energy requirements. The upcoming system can choose supercapacitor for this purpose.As wired communication protocol increases complexity wireless technologies such as Bluetooth and zigbee can be used for human robot interaction.

\section{REFERENCES}

[1] J. L. Pons, Ed., Wearable Robots: Biomechatronic Exoskeletons. New York:Wiley, Apr. 2008.

[2] Seyfarth, A., Grimmer, S., Maus, M., Häuflfle, D., Peuker, F., and Kalveram, K.-T. (2013). Biomechanical and neuromechanical concepts for legged locomotion.
Routledge Handbook of Motor Control and Motor Learning , 90-112

[3] Inertia Compensation Control of a One-Degree-of Freedom Exoskeleton for Lower-Limb Assistance: Initial Experiments Gabriel Aguirre-Ollinger, Member, IEEE

[4] IEEE transactions on neural systems and rehabilitation engineering, vol. 20, no. 1, January 2012

[5] Delp, S.L., Anderson, F.C., Arnold, A.S., Loan, P., Habib, A., John, C.T., Guendelman, E. Thelen,D.G.OpenSim:Open-source software to create and analyze dynamic simulations of movement. IEEE Transactions on Biomedical Engineering, vol. 55, pp. 19401950, 2007

[6] Copilusi C, Ceccarelli M, Carbone G, et al. Mechanism of a leg exoskeleton for walking rehabilitation purposes. In: New advances in mechanisms, transmissions and applications, Vol. 17, 2014, pp. 107-114. Dordrecht: Springer.

[7] Copilusi C, Ceccarelli M, Dumitru N, et al. Design and simulation of a leg exoskeleton linkage for a human rehabilitation system. In: The 11th IFToMM international symposium on science of mechanisms and machines, 2014, pp. 117-125. Cham: Springer

[8] Kavanagh JJ, Morrison S, James DA, et al. Reliability of segmental accelerations measured using a new wireless gait analysis system. J Biomech 2006; 39(15): 2863-2872.

[9] Singla A, Rupal BS and Virk GS. Optimization of stepped cone CVT for lower-limb exoskeletons. Perspect Sci 2016; 8:592-595.

[10] M. T. Smith and G. D. Baer, "Achievement of simple mobility milestones after stroke," Arch. Phys. Med. Rehabil., vol. 80, pp. 442-447, 1999. 